\title{
Instability of an elliptical flow: weakly nonlinear analysis
}

\author{
Y. Hattori ${ }^{1}$, Y. Fukumoto ${ }^{2} \&$ K. Fujimura ${ }^{3}$ \\ ${ }^{1}$ Division of Computer Aided Science, \\ Kyushu Institute of Technology, Japan \\ ${ }^{2}$ Graduate School of Mathematics and Space Environment Research \\ Center, Kyushu University, Japan \\ ${ }^{3}$ Department of Applied Mathematics and Physics, \\ Tottori University, Japan
}

\begin{abstract}
Elliptical flows are of interest since they appear in various vortical flows in which circular vortices are deformed by straining flow. We study the nonlinear evolution of an elliptical flow by weakly nonlinear analysis. Two sets of amplitude equations are derived for different situations. First, the evolution of bending waves is considered. Nonlinear interaction of the two base Kelvin waves results in cubic nonlinear terms, which leads to saturation of the elliptical instability. Next, the secondary instability is considered. Three Kelvin waves, one of which is a bending wave, form a resonant triad thanks to freedom of wavenumber shift. As a result three-wave equations augmented with linear terms are obtained as amplitude equations. They explain the previous numerical results on the secondary instability obtained by Kerswell J. Fluid Mech., 382, pp. 283-386, 1999.

Keywords: elliptical flow, weakly nonlinear analysis, triad resonance, secondary instability.
\end{abstract}

\section{Introduction}

Large-scale coherent vortical structures are ubiquitous in turbulent flows. They are often seen to destabilize and collapse to small-scale disorder. There are several important mechanisms of the destabilization among which are the elliptical instability [1-5] and the curvature instability [6, 7]. Both instabilities are the consequences of parametric resonance of two Kelvin waves mediated by 
symmetry-breaking perturbation. In the elliptical instability an imposed straining flow resonates Kelvin waves while in the curvature instability the self-induced flow due to the curvature of the vortex tube resonates them. These instabilities are well understood in the framework of linear stability analysis. However, linear theory is insufficient to explain the fast development of complicated fine-scale structures.

Weakly nonlinear analysis is a useful tool for studying the evolution of unstable waves beyond linear theory. Although limited to the range of weak nonlinearity, several important results are derived by it. For example, we can use it to find the type of bifurcation and the saturation amplitude (if the bifurcation is supercritical).

In this paper, we study the nonlinear evolution of an elliptical flow by weakly nonlinear analysis. Two sets of amplitude equations are derived for different situations. First, the nonlinear evolution of bending waves caused by selfinteraction is considered. The resulting nonlinear terms are cubic. Next, triad interaction is considered. Three Kelvin waves, one of which is a bending wave, form a triad thanks to finiteness of unstable wavenumber range. The resulting amplitude equations are three-wave equations augmented with linear terms. The results would be useful not only in analyzing transition to turbulence but also in studying the statistical properties of turbulence by using low-dimensional dynamical systems.

\section{Linear instability}

In this section we briefly summarize the results on the linear instability of an elliptical flow. Throughout the paper the flow is assumed to be inviscid and incompressible, though allowance may be made for small viscosity [3].

The base flow $\boldsymbol{U}$ is set to the sum of rigid body rotation and plain strain inside an elliptical cylinder. In a cylindrical coordinate system $(r, \theta, z)$, the velocity field $(U, V, W)$ and the pressure $P$ of the base flow is expressed as

$$
\begin{aligned}
\boldsymbol{U} & =\boldsymbol{U}_{0}+\varepsilon \boldsymbol{U}_{1}, \\
U_{0} & =W_{0}=0, \quad V_{0}=r, \quad P_{0}=r^{2} / 2-1, \\
U_{1} & =r \sin 2 \theta, \quad V_{1}=r \cos 2 \theta, \quad W_{1}=0, \quad P_{1}=0 .
\end{aligned}
$$

The boundary of the cylinder is $r=1-\varepsilon / 2 \cos 2 \theta$ at which slip condition is imposed. The total base flow has elliptical streamlines. The magnitude of strain $\varepsilon$ is assumed to be small but finite.

We add an infinitesimal disturbance $\boldsymbol{u}$ to the base flow. Perturbation expansion is legitimate for $\boldsymbol{u}$

$$
\boldsymbol{u}=\boldsymbol{u}_{01}+\varepsilon \boldsymbol{u}_{11}+\cdots
$$

The leading-order disturbance is set to the following type

$$
\boldsymbol{u}_{01}=\sum_{m} \boldsymbol{u}_{01}^{(m)}(r) \mathrm{e}^{\mathrm{i}\left(m \theta+k_{0} z-\omega_{0} t\right)} .
$$


Then the linearized Euler equation for $\boldsymbol{u}_{01}^{(m)}$ takes the following form

$$
\left[-\mathrm{i} \omega_{0} \mathrm{~L}+\mathrm{M}\left(m, k_{0}\right)\right] \boldsymbol{u}_{01}^{(m)}=0,
$$

where

$$
\mathrm{L}=\left(\begin{array}{cccc}
1 & 0 & 0 & 0 \\
0 & 1 & 0 & 0 \\
0 & 0 & 1 & 0 \\
0 & 0 & 0 & 0
\end{array}\right), \quad \mathrm{M}(m, k)=\left(\begin{array}{cccc}
\mathrm{i} m & -2 & 0 & \frac{\mathrm{d}}{\mathrm{d} r} \\
2 & \mathrm{i} m & 0 & \frac{\mathrm{i} m}{r} \\
0 & 0 & \mathrm{i} m & \mathrm{i} k \\
\frac{\mathrm{d}}{\mathrm{d} r}+\frac{1}{r} & \frac{\mathrm{i} m}{r} & \mathrm{i} k & 0
\end{array}\right) .
$$

In order to have non-trivial solutions the operator $-\mathrm{i} \omega_{0} \mathrm{~L}+\mathrm{M}\left(m, k_{0}\right)$ should be degenerate. This leads to dispersion relations between $\omega_{0}$ and $k_{0}$ (fig. 1). The solutions are the well-known Kelvin waves, which are neutrally stable.

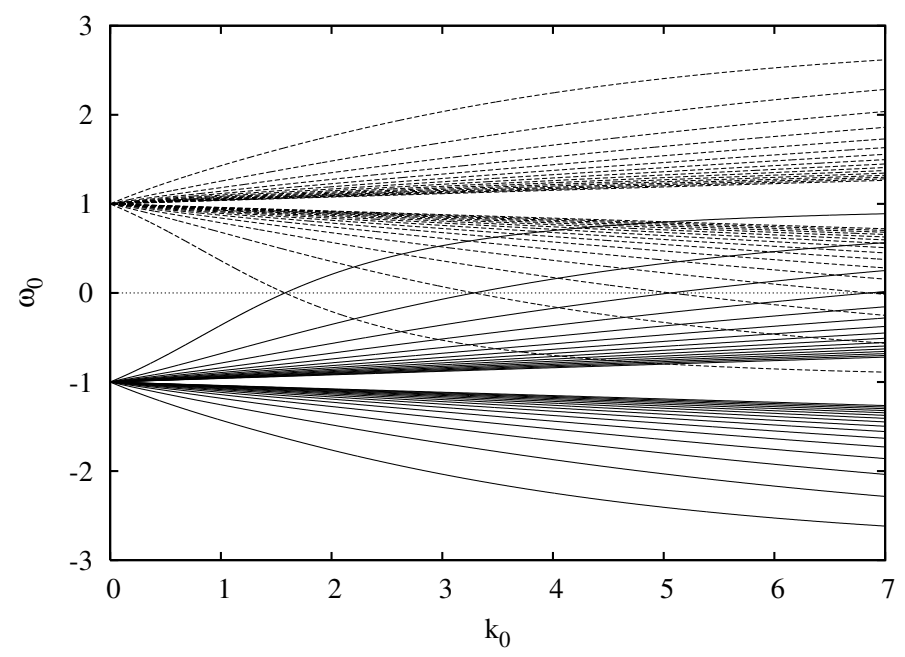

Figure 1: Dispersion relations of Kelvin waves: $m= \pm 1$.

Since the straining flow $\boldsymbol{U}_{1}$ has wavenumber two in $\theta$, a pair of Kelvin waves whose wavenumber $m$ differ by two can lead to parametric instability. In this case $\omega_{0}$ and $k_{0}$ should be a cross point of the dispersion curves of the two Kelvin waves. We focus on stationary bending waves: $m= \pm 1, \omega_{0}=0$. We set

$$
\boldsymbol{u}_{01}=A_{+} \boldsymbol{u}_{01}^{(1)}(r) \mathrm{e}^{\mathrm{i}\left(\theta+k_{0} z\right)}+\overline{A_{-}} \boldsymbol{u}_{01}^{(-1)}(r) \mathrm{e}^{\mathrm{i}\left(-\theta+k_{0} z\right)}
$$

(complex conjugate of $A_{-}$is introduced for convenience). Then the equations for $\boldsymbol{u}_{11}$ have forcing terms

$$
\mathrm{M}\left(1, k_{0}\right) \boldsymbol{u}_{11}^{(1)}=\overline{A_{-}} \mathrm{N}^{+}(-1) \boldsymbol{u}_{01}^{(-1)}, \quad \mathrm{M}\left(-1, k_{0}\right) \boldsymbol{u}_{11}^{(-1)}=A_{+} \mathrm{N}^{-}(1) \boldsymbol{u}_{01}^{(1)} .
$$


Since the above equations are singular, compatibility conditions force the amplitude of the Kelvin waves to vary slowly with time scale $\varepsilon^{-1}$ so that eqn. (5) is made solvable

$$
\begin{gathered}
\mathrm{M}\left(1, k_{0}\right) \boldsymbol{u}_{11}^{(1)}=\overline{A_{-}} \mathrm{N}^{+}(-1) \boldsymbol{u}_{01}^{(-1)}-\frac{\mathrm{d} A_{+}}{\mathrm{d} \tau} \mathrm{L} \boldsymbol{u}_{01}^{(1)}, \\
\mathrm{M}\left(-1, k_{0}\right) \boldsymbol{u}_{11}^{(-1)}=A_{+} \mathrm{N}^{-}(1) \boldsymbol{u}_{01}^{(1)}-\frac{\mathrm{d} \overline{A_{-}}}{\mathrm{d} \tau} \mathrm{L} \boldsymbol{u}_{01}^{(-1)},
\end{gathered}
$$

where $\tau=\varepsilon t$ and $\mathrm{N}^{+}=\overline{\mathrm{N}^{-}}$is defined by

$$
\mathrm{N}^{+}(m)=\frac{1}{2}\left(\begin{array}{cccc}
\mathrm{i}(1-m)+\mathrm{i} r \frac{\mathrm{d}}{\mathrm{d} r} & 0 & 0 & 0 \\
-2 & \mathrm{i}(-1-m)+\mathrm{i} r \frac{\mathrm{d}}{\mathrm{d} r} & 0 & 0 \\
0 & 0 & -\mathrm{i} m+\mathrm{i} r \frac{\mathrm{d}}{\mathrm{d} r} & 0 \\
0 & 0 & 0 & 0
\end{array}\right) .
$$

Closed form solutions are available [5]. Finally we arrive at

$$
\frac{\mathrm{d} A_{+}}{\mathrm{d} \tau}=\mathrm{i} a \overline{A_{-}}, \quad \frac{\mathrm{d} A_{-}}{\mathrm{d} \tau}=\mathrm{i} a \overline{A_{+}} .
$$

For the present case, a turns out to be real so that the system is unstable with exponential growth rate $|a|$. In general the stability of the above system is determined by the sign of energy of the two Kelvin waves: a pair of positive- and negative-energy waves or of zero-energy waves leads to instability [5].

\section{Weakly nonlinear analysis}

\subsection{General procedure}

Let us expand the total velocity field $\boldsymbol{u}_{T}$ in both shear strength $\varepsilon$ and disturbance amplitude $\alpha$

$$
\begin{aligned}
\boldsymbol{u}_{T}= & \boldsymbol{U}_{0}+\varepsilon \boldsymbol{U}_{1} \\
& +\alpha \boldsymbol{u}_{01}+\alpha^{2} \boldsymbol{u}_{02}+\alpha^{3} \boldsymbol{u}_{03}+\cdots+\varepsilon \alpha \boldsymbol{u}_{11}+\varepsilon \alpha^{2} \boldsymbol{u}_{12}+\cdots
\end{aligned}
$$

Similar expansions are assumed for pressure $p_{T}$. Note that $\boldsymbol{u}_{i 1}$ in (1) corresponds to $\alpha \boldsymbol{u}_{i 1}$ in (7).

We substitute (7) into the Euler equations

$$
\begin{aligned}
\frac{\partial \boldsymbol{u}_{T}}{\partial t}+\boldsymbol{u}_{T} \cdot \boldsymbol{\nabla} \boldsymbol{u}_{T} & =-\nabla p_{T}, \\
\nabla \cdot \boldsymbol{u}_{T} & =0 .
\end{aligned}
$$

At each order $O\left(\varepsilon^{i} \alpha^{j}\right)$ we obtain linear equations. Depending on the combination of modes in the leading-order disturbance $\boldsymbol{u}_{01}$, solvability conditions force the amplitude of the modes to vary slowly, which derives amplitude equations [8]. 


\subsection{Nonlinear saturation of the linear instability}

First, we consider nonlinear evolution of bending waves $\boldsymbol{u}_{01}=A_{+} \mathrm{e}^{\mathrm{i}\left(\theta+k_{0} z\right)}+$ $A_{-} \mathrm{e}^{\mathrm{i}\left(\theta-k_{0} z\right)}+$ c.c., due to self-induced nonlinearity.

At $O\left(\alpha^{2}\right)$ the equation is

$$
\begin{aligned}
\frac{\partial \boldsymbol{u}_{02}}{\partial t}+\boldsymbol{U}_{0} \cdot \boldsymbol{\nabla} \boldsymbol{u}_{02}+\boldsymbol{\nabla} p_{02} & =-\boldsymbol{u}_{01} \cdot \boldsymbol{\nabla} \boldsymbol{u}_{01}, \\
\boldsymbol{\nabla} \cdot \boldsymbol{u}_{02} & =0 .
\end{aligned}
$$

The solution is in the form

$$
\begin{aligned}
\boldsymbol{u}_{02}= & A_{+}^{2} \mathrm{e}^{\mathrm{i}\left(2 \theta+2 k_{0} z\right)} \boldsymbol{u}_{A_{+} A_{+}}+A_{-}^{2} \mathrm{e}^{\mathrm{i}\left(2 \theta-2 k_{0} z\right)} \boldsymbol{u}_{A_{-} A_{-}} \\
& +A_{+} A_{-} \mathrm{e}^{\mathrm{i} 2 \theta} \boldsymbol{u}_{A_{+} A_{-}}+A_{+} \overline{A_{-}} \mathrm{e}^{\mathrm{i} 2 k_{0} z} \boldsymbol{u}_{A_{+}} \overline{A_{-}}+\text {c.c. } \\
& +\left|A_{+}\right|^{2} \boldsymbol{u}_{\left|A_{+}\right|^{2}}+\left|A_{-}\right|^{2} \boldsymbol{u}_{\left|A_{-}\right|^{2}},
\end{aligned}
$$

where $\boldsymbol{u}_{\left|A_{ \pm}\right|^{2}}$ is shown to vanish.

At $O\left(\alpha^{3}\right)$ the equation is

$$
\begin{aligned}
\frac{\partial \boldsymbol{u}_{03}}{\partial t}+\boldsymbol{U}_{0} \cdot \boldsymbol{\nabla} \boldsymbol{u}_{03}+\boldsymbol{\nabla} p_{03} & =-\boldsymbol{u}_{01} \cdot \boldsymbol{\nabla} \boldsymbol{u}_{02}-\boldsymbol{u}_{02} \cdot \boldsymbol{\nabla} \boldsymbol{u}_{01}, \\
\boldsymbol{\nabla} \cdot \boldsymbol{u}_{03} & =0 .
\end{aligned}
$$

The solution is in the form

$$
\begin{aligned}
\boldsymbol{u}_{03}= & \left(\left|A_{+}\right|^{2} A_{+} \boldsymbol{u}_{\left|A_{+}\right|^{2} A_{+}}+\left|A_{-}\right|^{2} A_{+} \boldsymbol{u}_{\left|A_{-}\right|^{2} A_{+}}\right) \mathrm{e}^{\mathrm{i}\left(\theta+k_{0} z\right)} \\
& +\left(\left|A_{+}\right|^{2} A_{-} \boldsymbol{u}_{\left|A_{+}\right|^{2} A_{-}}+\left|A_{-}\right|^{2} A_{-} \boldsymbol{u}_{\left|A_{-}\right|^{2} A_{-}}\right) \mathrm{e}^{\mathrm{i}\left(\theta-k_{0} z\right)} \\
& + \text { c.c. }+ \text { (irrelevant terms) }
\end{aligned}
$$

Here only the terms leading to compatibility conditions are written explicitly. Since the equations for $\boldsymbol{u}_{\left|A_{+}\right|^{2} A_{+}}$etc. are singular, the amplitudes $A_{ \pm}$should vary slowly with time scale $\alpha^{-2}$. Mean flow correction should be also regarded; it arises at $O\left(\varepsilon \alpha^{2}\right)$.

By setting $\varepsilon=\alpha^{2}$ we can derive the following set of amplitude equations

$$
\begin{aligned}
\frac{\mathrm{d} A_{+}}{\mathrm{d} \tau} & =\mathrm{i} a \overline{A_{-}}-\mathrm{i}\left(c\left|A_{-}\right|^{2}+d C\right) A_{+}, \\
\frac{\mathrm{d} A_{-}}{\mathrm{d} \tau} & =\mathrm{i} a \overline{A_{+}}-\mathrm{i}\left(c\left|A_{+}\right|^{2}+d C\right) A_{-}, \\
\frac{\mathrm{d} C}{\mathrm{~d} \tau} & =\mathrm{i}\left(A_{+} A_{-}-\overline{A_{+} A_{-}}\right),
\end{aligned}
$$

where $C$ is the amplitude of the mean flow correction and the coefficients $c$ and $d$ are determined by the solvability conditions at $O\left(\alpha^{3}\right)$ and $O\left(\varepsilon \alpha^{2}\right)$, respectively. It is worth noting that the terms proportional to $\left|A_{+}\right|^{2} A_{+}$and $\left|A_{-}\right|^{2} A_{-}$vanish. Saturation of elliptical instability is explained by the equations above. 


\subsection{Secondary instability via triad resonance}

Next, we consider nonlinear evolution due to triad resonance. Once bending waves are established, they can be regarded as perturbation which could induce another instability (secondary instability). Kerswell [9] showed that this is indeed the case for rapidly rotating flow without strain by numerical linear stability analysis; the instability is most likely due to triad resonances of the Kelvin waves.

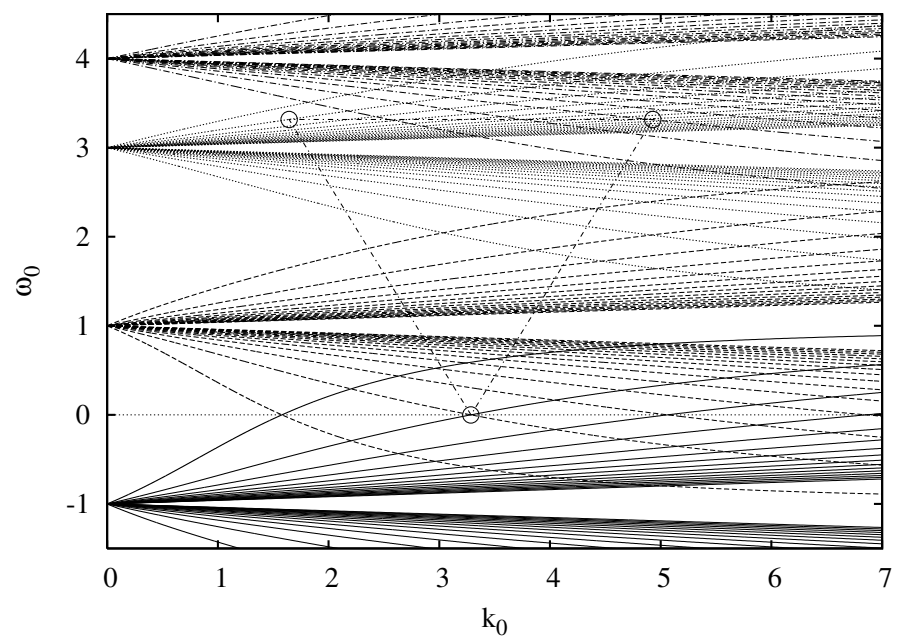

Figure 2: Dispersion relations of Kelvin waves and triad: $m= \pm 1,3$ and 4 .

Although it is difficult to find exact resonant triads of neutral Kelvin waves, the elliptical instability allows $O(\varepsilon)$-margin of wavenumber of the unstable bending modes so that triads are exactly formed. In fig. 2 we show one of these triads: $(m ; k, \omega)=\left(1 ; k_{*}, 0\right),\left(3 ; k_{*} / 2, \omega_{0}\right),\left(4 ; 3 k_{*} / 2, \omega_{0}\right)$, where $k_{*} \approx 3.273$ and $\omega_{0} \approx 3.32$. In fact, the wavenumber of the corresponding neutral bending mode ( $m=1)$ is $k_{0} \approx 3.286$. It shifts generally as $k=k_{0}+\varepsilon k_{1}$ for the elliptical flow; the bending wave is destabilized for $\left|k-k_{0}\right| \leq \varepsilon \Delta k$ so that we have $k_{*}=k_{0}+\varepsilon k_{1}$ for $\varepsilon \geq \varepsilon_{c}=\left|k_{*}-k_{0}\right| / \Delta k$.

Let us set

$$
\begin{aligned}
\boldsymbol{u}_{01}= & A_{+} \mathrm{e}^{\mathrm{i}\left(\theta+k_{*} z\right)}+B_{+} \mathrm{e}^{\mathrm{i}\left(3 \theta+k_{*} z / 2-\omega_{0} t\right)}+C_{+} \mathrm{e}^{\mathrm{i}\left(4 \theta+3 k_{*} z / 2-\omega_{0} t\right)} \\
& +A_{-} \mathrm{e}^{\mathrm{i}\left(\theta-k_{*} z\right)}+B_{-} \mathrm{e}^{\mathrm{i}\left(3 \theta-k_{*} z / 2-\omega_{0} t\right)}+C_{-} \mathrm{e}^{\mathrm{i}\left(4 \theta-3 k_{*} z / 2-\omega_{0} t\right)}+\text { c.c. }
\end{aligned}
$$

Then compatibility conditions arise at $O\left(\alpha^{2}\right)$. By setting $\varepsilon=\alpha$ we can derive the following set of amplitude equations

$$
\frac{\mathrm{d} A_{ \pm}}{\mathrm{d} \tau}=\mathrm{i} \beta_{1} \overline{A_{\mp}}+\mathrm{i} \beta_{2} A_{ \pm}+\mathrm{i} \gamma_{1} \overline{B_{ \pm}} C_{ \pm},
$$




$$
\frac{\mathrm{d} B_{ \pm}}{\mathrm{d} \tau}=\mathrm{i} \gamma_{2} \overline{A_{ \pm}} C_{ \pm}, \quad \frac{\mathrm{d} C_{ \pm}}{\mathrm{d} \tau}=\mathrm{i} \gamma_{3} A_{ \pm} B_{ \pm} .
$$

The coefficients are evaluated by

$$
\begin{aligned}
\mathrm{i} \beta_{1}\left\langle\boldsymbol{u}_{A_{+}} \mid \mathrm{L} \boldsymbol{u}_{A_{+}}\right\rangle= & \left\langle\boldsymbol{u}_{A_{+}} \mid \mathrm{N}^{+}(1) \overline{\boldsymbol{u}}_{A_{-}}\right\rangle-\left(\frac{1}{4} \frac{\partial \bar{u}_{A_{-}}}{\partial r}-\frac{\mathrm{i}}{2} \bar{v}_{A_{-}}\right) p_{A_{+}}, \\
\beta_{2}\left\langle\boldsymbol{u}_{A_{+}} \mid \mathrm{L} \boldsymbol{u}_{A_{+}}\right\rangle= & -k_{1}\left\langle\boldsymbol{u}_{A_{+}} \mid \mathrm{W} \boldsymbol{u}_{A_{+}}\right\rangle \\
\mathrm{i} \gamma_{1}\left\langle\boldsymbol{u}_{A_{+}} \mid \mathrm{L} \boldsymbol{u}_{A_{+}}\right\rangle= & \left\langle\boldsymbol{u}_{A_{+}} \mid\left\{\overline{\boldsymbol{u}}_{B_{+}}, \boldsymbol{u}_{C_{+}}, 4,3 k_{0} / 2\right\}\right\rangle \\
& +\left\langle\boldsymbol{u}_{A_{+}} \mid\left\{\boldsymbol{u}_{C_{+}}, \overline{\boldsymbol{u}}_{B_{+}},-3,-k_{0} / 2\right\}\right\rangle \\
\mathrm{i} \gamma_{2}\left\langle\boldsymbol{u}_{B_{+}} \mid \mathrm{L} \boldsymbol{u}_{B_{+}}\right\rangle= & \left\langle\boldsymbol{u}_{B_{+}} \mid\left\{\bar{u}_{A_{+}}, \boldsymbol{u}_{C_{+}}, 4,3 k_{0} / 2\right\}\right\rangle \\
& +\left\langle\boldsymbol{u}_{B_{+}} \mid\left\{\boldsymbol{u}_{C_{+}}, \overline{\boldsymbol{u}}_{A_{+}},-1,-k_{0}\right\}\right\rangle \\
\mathrm{i} \gamma_{3}\left\langle\boldsymbol{u}_{C_{+}} \mid \mathrm{L} \boldsymbol{u}_{C_{+}}\right\rangle= & \left\langle\boldsymbol{u}_{C_{+}} \mid\left\{\boldsymbol{u}_{A_{+}}, \boldsymbol{u}_{B_{+}}, 3, k_{0} / 2\right\}\right\rangle \\
& +\left\langle\boldsymbol{u}_{C_{+}} \mid\left\{\boldsymbol{u}_{B_{+}}, \boldsymbol{u}_{A_{+}}, 1, k_{0}\right\}\right\rangle
\end{aligned}
$$

where

$$
\begin{aligned}
\left\langle\boldsymbol{u}_{X}, \boldsymbol{u}_{Y}\right\rangle & =\int_{0}^{1}\left(\bar{u}_{X} u_{Y}+\overline{v_{X}} v_{Y}+\overline{w_{X}} w_{Y}+\overline{p_{X}} p_{Y}\right) r \mathrm{~d} r \\
\mathrm{~W} & =\left(\begin{array}{cccc}
0 & 0 & 0 & 0 \\
0 & 0 & 0 & 0 \\
0 & 0 & 0 & 1 \\
0 & 0 & 1 & 0
\end{array}\right), \\
\left\{\boldsymbol{u}_{X}, \boldsymbol{u}_{Y}, m, k\right\} & =-\left(\begin{array}{l}
{\left[u_{X} \frac{\mathrm{d}}{\mathrm{d} r}+\frac{\mathrm{i} m v_{X}}{r}+\mathrm{i} k w_{X}\right] u_{Y}-\frac{v_{X} v_{Y}}{r}} \\
\left.u_{X} \frac{\mathrm{d}}{\mathrm{d} r}+\frac{\mathrm{i} m v_{X}}{r}+\mathrm{i} k w_{X}\right] v_{Y}+\frac{v_{X} u_{Y}}{r} \\
{\left[u_{X} \frac{\mathrm{d}}{\mathrm{d} r}+\frac{\mathrm{i} m v_{X}}{r}+\mathrm{i} k w_{X}\right] w_{Y}}
\end{array}\right) .
\end{aligned}
$$

An example of the evolution of the mode energies is shown in fig. 3. Initially the energy of bending modes $E_{A \pm}=\left|A_{ \pm}\right|^{2}$ is set to large values compared to $E_{B \pm}$ and $E_{C \pm}$. Exponential increase of $E_{B+}$ and $E_{C+}$, which corresponds to the secondary instability, is observed. The mode energy $E_{A+}$ almost vanishes and $E_{B+}$ and $E_{C+}$ saturate around $t=9.5$; then $E_{B+}$ and $E_{C+}$ decrease exponentially. In the exponential increase or decrease, the ratio of mode energies is easily evaluated as $E_{B+} / E_{C+}=\left|\gamma_{2} / \gamma_{3}\right| \approx 0.46$, which is comparable to the value 0.545 in Kerswell [9]. In the example the mode energies evolve periodically; this is not expected in the actual flow, however. Conceivably, the secondary instability would be taken over, at some stage, by a tertiary and higher instabilities as implied by the Hamiltonian normal form $[10,11]$. 


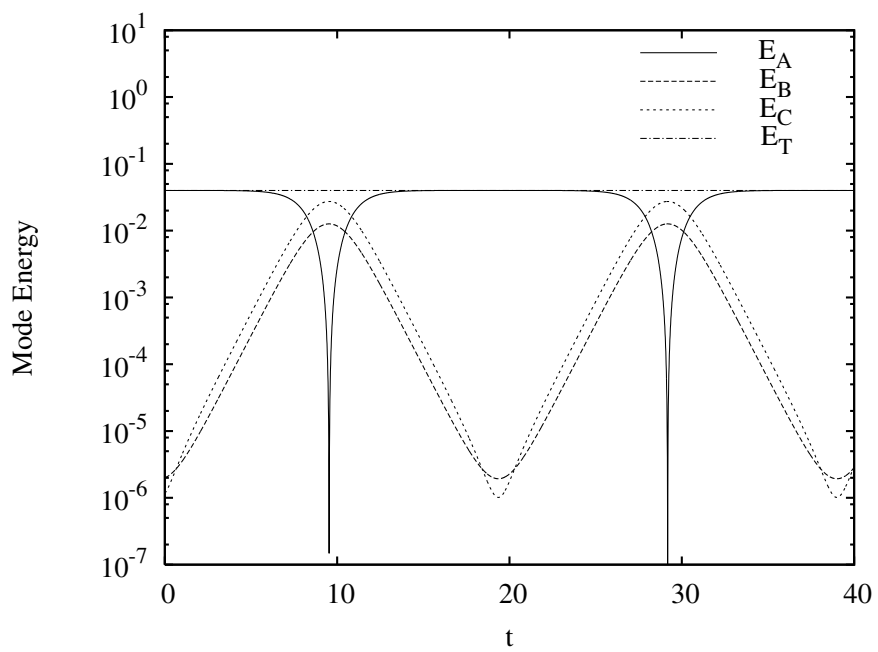

Figure 3: Evolution of mode energies - Resonant triad.

\section{Conclusion}

The evolution of an elliptical flow is studied by weakly nonlinear analysis. Two sets of amplitude equations are derived for different situations: the first one can be used to estimate saturation amplitude of destabilized bending waves due to self-induced nonlinear effect; the second one shows that triad interaction is responsible for the secondary instability found numerically by Kerswell [9]. The results would be useful in understanding the nonlinear evolution of elliptical flows. Direct numerical simulation, which is planned as a future work, should assess the applicability of the present analysis and reveal other processes important in the fully nonlinear evolution.

\section{References}

[1] Moore, D. W. \& Saffman, P. G., The instability of a straight vortex filament in a strain field. Proc. R. Soc. Lond. A, 346, pp. 413-425, 1975.

[2] Tsai, C.-Y. \& Widnall, S. E., The stability of short waves on a straight vortex filament in a weak externally imposed strain field. J. Fluid Mech., 73, pp. 721-733, 1976.

[3] Eloy, C. \& Le Dizès, S., Stability of the Rankine vortex in a multipolar strain field. Phys. Fluids, 13, pp. 660-676, 2001.

[4] Kerswell, R. R., Elliptical instability. Annu. Rev. Fluid Mech., 34, pp. 83113, 2002.

[5] Fukumoto, Y., The three-dimensional instability of a strained vortex tube revisited. J. Fluid Mech., 493, pp. 287-318, 2003. 
[6] Hattori, Y. \& Fukumoto, Y., Short-Wavelength Stability Analysis of Thin Vortex Rings. Phys. Fluids, 15 pp. 3151-3163, 2003.

[7] Fukumoto, Y. \& Hattori, Y., Curvature Instability of a Vortex Ring. J. Fluid Mech., 526, pp. 77-115, 2005.

[8] Sipp, D., Weakly nonlinear saturation of short-wave instabilities in a strained Lamb-Oseen vortex. Phys. Fluids, 12, pp. 1715-1729, 2000.

[9] Kerswell, R. R., Secondary instabilities in rapidly rotating fluids: inertial wave breakdown. J. Fluid Mech., 382, pp. 283-386, 1999.

[10] Guckenheimer, J. \& Mahalov, A., Instability induced by symmetry reduction. Phys. Rev. Lett., 68, pp. 2257-2260, 1992.

[11] Knobloch, E., Mahalov, A. \& Marsden, J. E., Normal forms for threedimensional parametric instabilities in ideal hydrodynamics. Physica $D, \mathbf{7 3}$, pp. 49-81, 1994. 